\title{
Overview Of The R\&D Activities For The ITER Bolometer Diagnostic
}

\author{
Hans Meister $^{* 1}$, H. Eixenberger ${ }^{1}$, C. Gliss ${ }^{1}$, A. Kampke ${ }^{1}$, M. Kannamüller ${ }^{1}$, J. Koll ${ }^{1}$, F.

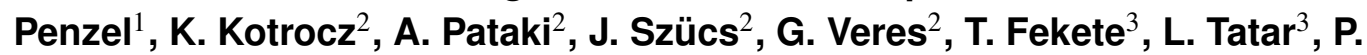 \\ Detemple $^{4}$, K. Mpoukouvalas ${ }^{4}$ and S. Schmitt ${ }^{4}$ \\ ${ }^{1}$ Max-Planck-Institute for Plasmaphysics, Boltzmannstr. 2, 85748 Garching, Germany \\ ${ }^{2}$ Wigner Research Centre for Physics, Konkoly Thege Miklós út 29-33, H-1121 Budapest, \\ Hungary \\ ${ }^{3}$ Centre for Energy Research, Hungarian Academy of Sciences, Konkoly Thege Miklós út 29-33, \\ H-1121 Budapest, Hungary \\ ${ }^{4}$ Fraunhofer ICT-IMM, Carl-Zeiss-Str. 18-20, 55129 Mainz, Germany \\ E-mail: meister@ipp.mpg.de
}

\begin{abstract}
The detailed development of the ITER bolometer diagnostic has recently been started. The aim is to reach a level from which on built-to-print drawings for the diagnostic components can be produced. Based on the challenges and corresponding requirements a review of the development status reveals that for some issues solutions are available. Among them are the damping of ECRH stray radiation, precise definition of the geometry of narrow lines-of-sight and their in-situ measurement, first prototype components for operation at high temperatures as well as detector prototypes with thick absorbers to detect photon energies up to $20 \mathrm{keV}$ and which are potentially radiation resistant. However, there are also several issues still to be solved. Prominent among those discussed is the mechanical stability of the detector prototypes at high operating temperatures, but also the detailed definition of bolometer camera components suited for the integration in ITER and capable to withstand the operating conditions, in particular the effects of neutrons. A development path is proposed which focuses on the open issues and includes proposals for crucial prototype tests to validate design concepts.
\end{abstract}

1st EPS conference on Plasma Diagnostics

14-17 April 2015

Frascati, Italy

\footnotetext{
* Speaker.
} 


\section{Introduction}

The total radiated power as well as the radiation emission profile on ITER will be determined by the bolometer diagnostic. A bolometer measures the plasma radiation over a wide spectral range (from soft-X to IR) by monitoring the temperature rise induced by deposition of photon energy in the absorber of the bolometer. The reference detector type chosen for ITER is the miniaturised metal resistor bolometer, which uses a metallic meander as temperature sensor [1,2]. The spatial distribution of the emission profile can be determined using many lines-of-sight (LOS) and by applying tomographic reconstruction techniques.

The objective of ITER is to produce net fusion power and thus a significant amount of high energy neutrons. All components of the bolometer diagnostic, including the sensors, will therefore be subject to requirements, environmental conditions and loads in ITER which surpass those of currently operating devices. A first step in the decision on the most suitable development path is to summarise and prioritise the requirements to ensure that the $R \& D$ activities meet the demands. The most important ones for the bolometer diagnostic are given in section 2. Sections 3 and 4 discuss the status of $R \& D$ as well as further required development for the in-vessel diagnostic components and the bolometer sensor, respectively.

\section{Requirements for bolometer components in ITER}

The most stringent requirements are related to ITER being a nuclear device operating in high vacuum at high temperatures. Thus, all materials used to manufacture the diagnostic components, which are distributed over all of the vacuum vessel, in divertor cassettes and in port plugs, must be vacuum compatible and able to withstand the high fluence of neutrons and secondary gamma emission. As some bolometer cameras will be placed in the ITER vessel at locations without planned maintenance, this translates into a requirement on the life time of 20 years, the whole life time of ITER. The loads associated to the nuclear environment require the materials to be chosen such that potential effects like radiation damage or transmutation do not affect the functionality or significantly reduce the performance. Radiation damage for bolometers in ITER is expected to be up to 0.3 dpa [3], depending on location. Because of the high neutron fluence thermal loads of up to $2 \mathrm{MW} / \mathrm{m}^{3}$ have to be taken into account as well. In addition to these loads, the baking of the vessel, up to $350^{\circ} \mathrm{C}$ in the divertor cassettes, as well as stray radiation from the ECRH heating system in the mm-wavelength range have to be considered.

Furthermore, robustness is required by the diagnostic components in view of normal and offnormal environmental conditions. Such loads are pressure pulses during massive gas injection for mitigating disruptions, electro-magnetic loads and associated forces during disruptions, seismic loads and water and steam ingress due to water leaks.

The bolometer diagnostic is required to provide data with a time resolution of $1 \mathrm{~ms}$. This requires for the sensor that the heat transfer time from absorber to the meander is significantly lower in order not to compromise the evaluation of total radiated power. Alternatively, the time dependence of the heat pulse could be included in the evaluation, but the enhanced complexity would increase the uncertainty of the result. Furthermore, the absorber needs to provide a sufficient attenuation length for the expected photon energies. 


\section{Development of diagnostic components}

Figure 1(a) gives an overview of the main components of a typical collimator-type bolometer camera. The camera housing supports the detector within its holder, attaches it to the wall of the vacuum vessel or any other supporting structure and ensures good thermal conductivity to the wall for the thermal loads. The detector holder supports the sensor and provides means for connecting the signal cables. In front of the sensor is a collimator, which defines the geometry of the LOS using several apertures within it and the top plate as last aperture. On all locations in ITER cameras are placed behind large components for neutron shielding which leave only small gaps for viewing the plasma.

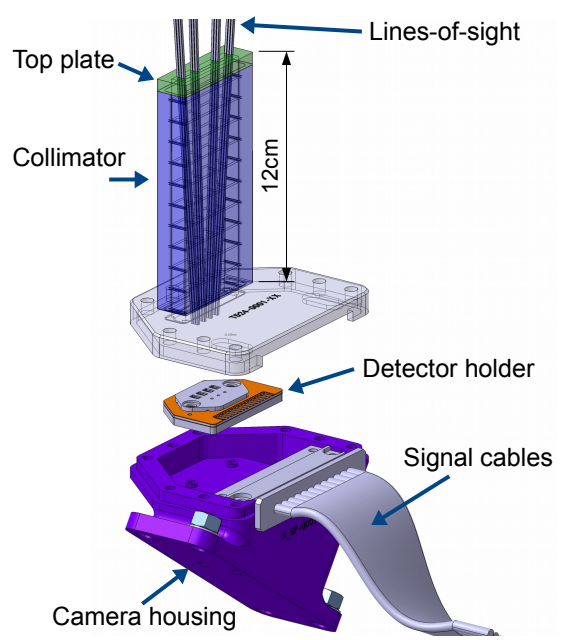

(a) Main components of a bolometer camera.

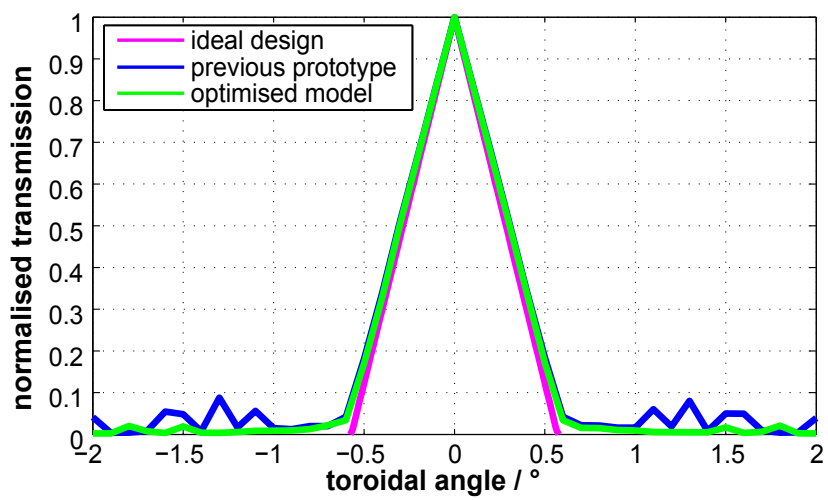

(b) MCRT simulation results for a collimator in toroidal direction.

Figure 1: Typical design of a bolometer collimator camera and MCRT results.

About 500 LOS are foreseen in ITER to cover the complete plasma cross section and provide the possibility of determining the spatial distribution of the plasma radiation profile. A prerequisite for a successful tomographic reconstruction is the knowledge about the transmission function for each LOS and a sufficient reduction of reflections and/or stray light [6]. To measure the transmission function of collimators and to prove the capability of a method for in situ measurement of LOS geometries, the test facility IBOROB has been brought into operation $[4,5]$.

Supporting Monte-Carlo ray-tracing (MCRT) calculations led to the definition of a procedure for determining the optimum design parameters of collimators with respect to geometry and stray light suppression [7]. Figure 1(b) shows the result of MCRT simulations for a non-optimised prototype (blue), which was confirmed by measurements using IBOROB and still shows impact of stray light, and an optimised collimator (green) in comparison to the ideal case as expected from the design parameters (pink). This demonstrates that even very narrow viewing cones with only $1^{\circ}$ total opening angle and acceptable stray light can be designed and manufactured.

In order to protect the sensor from ECRH stray radiation, dedicated components have been designed and tested. A combination of designing the apertures in the top plate as microwave-reflecting grid and coating the inside of the collimator channels with a microwave absorbing ceramic leads to an attenuation of at least $70 \mathrm{~dB}$ between $125-225 \mathrm{GHz}$ and about $40 \mathrm{~dB}$ for higher frequencies [8]. 
However, the complete bolometer camera needs to be designed microwave tight. In particular the feeding of the signal cables is to be developed and tested on prototypes.

Another generic issue for all cameras to be developed is the reliable connection of the signal cables to the sensor. The proposed technique is to use a ceramic printed circuit board (PCB) as detector holder and to place bond wires between sensor and PCB. The external signal cables can then be laser welded or hard soldered. To this aim, the bonding and welding methods need to be developed and tested in combination with the materials finally chosen for the sensor. The prototype tests will have to prove the reliable connection under radiation loads, too.

Additionally, further activities need to decide which camera type, collimator or pin-hole, is most suitable for individual locations in ITER. For each location the implications due to mechanical loads and stresses (EM and seismic forces, thermal loads and stresses) have to be analysed and the design options validated. A particular issue will be to optimise the individual camera designs based on the available construction space and gap sizes between in-vessel components. For the resulting designs high priority prototype tests have been identified to enable design choices and to verify the meeting of requirements. Most prominent among them are the radiation resistance of electrical connections. But also tests demonstrating the capability to withstand mechanical loads as well as those validating remote-handling capabilities need to be foreseen.

\section{Bolometer sensor developments}

The materials for absorber, substrate and meander influence the sensitivity of the sensor as well as its resistivity against loads. Initial developments used up to $7 \mu \mathrm{m}$ thick Au absorbers on a $\approx 20 \mu \mathrm{m}$ mica or kapton foil with Au meanders, as do the sensors operating in JET [9, 10]. New developments focused on $12.5 \mu \mathrm{m}$ Pt absorbers and meanders, supported by a $3 \mu \mathrm{m} \mathrm{SiN} \mathrm{mem-}$ brane $[2,11]$, which proved to be very sensitive [12]. Investigations on the irradiation resistance for the JET-type sensors have been carried out up to a fast neutron fluence of $0.1 \mathrm{dpa}$ within a fission-reactor energy spectrum [13]. An increase in meander resistance was found due to the transmutation of $\mathrm{Au}$ to $\mathrm{Hg}$. The post-irradiation examination confirmed that the $\mathrm{Au}$ meander contained $46 \%$ mercury. For an ITER-like neutron energy spectrum a transmutation rate of $\approx 10 \%$ is expected. The extent of swelling of the mica substrate was small $\left(0.13 \%\right.$ for $150^{\circ} \mathrm{C}$ and $\left.0.01 \mathrm{dpa}\right)$. Pt-SiN-Pt sensors were also investigated under neutron loads up to $0.013 \mathrm{dpa}$ and no degradation of the performance was observed [14]. However, after dismantling a fracture of the sensors and a delamination of meanders was found, probably due to the thermal loads during irradiation tests.

The aging of JET-type sensors by baking cycles at $160^{\circ} \mathrm{C}$ for $6 \mathrm{~h}$ showed no degradation of the properties [15]. Pt-SiN-Pt sensor prototypes were subjected to repetitive thermal cycling up to $450^{\circ} \mathrm{C}$ [16]. Their reliability at elevated operating temperatures is not yet sufficient because of material stresses and thermal cycling leading to a fatigue cycle caused by the different thermal expansion coefficients between SiN and Pt. This effect increses with absorber thickness and temperature.

The probability of high energy photons (above several keV) being absorbed by the bolometer sensor depends on the thickness and the mass attenuation coefficient of the material used for the absorber. The resulting fraction of absorbed total $P_{\text {rad }}$, as calculated using the approximation of a circular plasma shape for a volume integrated radiational power density, is given in Table 1. Thus, 
the bolometer diagnostic needs to consider absorber thicknesses in the range of $15 \mu \mathrm{m}$ to ensure that at least $90 \%$ of the total radiation can be detected.

\begin{tabular}{|c|c|c|c|c|c|}
\hline Thickness $/ \mu \mathrm{m}$ & 7 & 12 & 15 & 20 & 30 \\
\hline $\mathrm{Pt}$ & $84.3 \%$ & $89.5 \%$ & $91.1 \%$ & $92.6 \%$ & $94.6 \%$ \\
\hline $\mathrm{Au}$ & $83.5 \%$ & $88.9 \%$ & $90.6 \%$ & $92.4 \%$ & $94.3 \%$ \\
\hline
\end{tabular}

Table 1: Fraction of absorbed total radiation depending on thickness and material of a bolometer sensor for the ITER standard $15 \mathrm{MA}$ scenario with low impurity content $\left(Z_{\mathrm{eff}}=1.5\right)$

The time within which the bolometer sensor can provide a signal in response to the change of radiational power falling onto the absorber is determined by the heat transfer time $t_{h}$ from the absorber surface to the meander. For some material combinations $t_{h}$ has been calculated (see Table 2). Obviously, mica as substrate will not allow a direct achievement of the required time resolution.

\begin{tabular}{|l|l|l|l|}
\hline Temperature $/{ }^{\circ} \mathrm{C}$ & $\begin{array}{l}t_{h} \text { for } 14 \mu \mathrm{m} \mathrm{Au} \mathrm{on} \\
20 \mu \mathrm{m} \mathrm{Mica} / \mu \mathrm{s}\end{array}$ & $\begin{array}{l}t_{h} \text { for } 14 \mu \mathrm{m} \mathrm{Au} \mathrm{on} \\
3 \mu \mathrm{m} \mathrm{SiN} / \mu \mathrm{s}\end{array}$ & $\begin{array}{l}t_{h} \text { for } 12.5 \mu \mathrm{m} \mathrm{Pt} \text { on } \\
3 \mu \mathrm{m} \mathrm{SiN} / \mu \mathrm{s}\end{array}$ \\
\hline 25 & 1875 & 1.8 & 6.4 \\
\hline
\end{tabular}

Table 2: Heat transfer times calculated for three combinations of absorber and substrate material.

In order to develop a working bolometer sensor for ITER within the tight schedule constraints a development based on the advantages of the respective types is proposed to be conducted in parallel. The good mechanical stability at high temperatures of mica as substrate should be combined with Pt-meanders to eliminate resistance changes due to irradiation. Additionally, Au absorbers on thin SiN membranes with Pt meanders are also good candidates as Au is more ductile than Pt, thus leading to better stability and providing the opportunity to meet the time measurement requirements directly. The transmutation in the absorber does not deteriorate the performance.

An additional alternative would be to investigate flexure hinges as support for the absorber as in micro bolometers for IR cameras [17]. However, this mechanical reliable solution must prove that it can be used as bolometer with calibration parameters in the correct order of magnitude.

\section{Conclusion}

For a successful development of the ITER bolometer diagnostic parallel development paths seem unavoidable, in particular for the development of the sensor as currently no prototype meeting all requirements exists. R\&D activities so far managed to identify suitable materials and designs; subsequent activities have to investigate which proposed combination and design matches the requirements best. Similarly, the development of in-vessel components successfully led to solutions for dealing with ECRH stray radiation and to define extremely narrow viewing cones for the LOS as well as how to measure them in situ. In need of further R\&D and prototype testing are the electrical connections and significant detailed design for individual cameras will be required.

\section{Acknowledgements}

The authors greatfully acknowledge the input data for the plasma radiation in ITER provided 
by M. O'Mullane and the most valuable discussions and inputs on sensor development and risk assessment from C.I. Ingesson (F4E) and R. Reichle (ITER).

The work leading to this publication has been funded partially by F4E under Specific Grant Agreement F4E-FPA-384-SG01. This publication reflects the views only of the authors, and F4E cannot be held responsible for any use which may be made of the information contained therein.

\section{References}

[1] K. F. Mast et al. A low noise highly integrated bolometer array for absolute measurement of VUV and soft-X radiation. Rev. Sci. Instrum., 62(3):744-750, 1991.

[2] H. Meister et al. The ITER bolometer diagnostic - status and plans. Rev. Sci. Instrum., 79:10F511, 2008.

[3] A. Suarez et al. Neutronic analysis for bolometers in ITER. Fusion Eng. Des., 88(6-8):1395-1399, 2013.

[4] H. Meister et al. Development of an automated method for in-situ measurement of the geometrical properties of the ITER bolometer diagnostic. Fusion Eng. Des., 86:1170-1173, 2011.

[5] F. Penzel et al. Assessment of line of sight characteristics of ITER bolometer prototype collimators. Fusion Eng. Des., 88(6-8):1267-1270, 2013.

[6] L. C. Ingesson and D. J. Wilson. Optimization of apertures and collimators for multi-channel plasma diagnostics. Rev. Sci. Instrum., 73(8):2890-2899, 2002.

[7] N. Homner. Kollimatoroptimierung für die ITER-Bolometerdiagnostik mittels Monte-Carlo Raytracing. Bachelor's thesis, Ludwig-Maximilians-Universität München, 2014.

[8] H. Meister et al. Millimetre wave attenuation of prototype diagnostic components for the ITER bolometers. In $29^{\text {th }}$ Symposium on Fusion Technology (SOFT-29), San Sebastian, Spain, 2014.

[9] K. F. Mast et al. Bolometric diagnostic in JET. Rev. Sci. Instrum., 56(5):969-971, 1985.

[10] A. Huber et al. Upgraded bolometer system on JET for improved radiation measurements. Fusion Eng. Des., 82(5-14):1327-1334, 2007.

[11] H. Meister et al. Optimization of a bolometer detector for ITER based on Pt absorber on SiN membrane. Rev. Sci. Instrum., 81:10E132, 2010.

[12] H. Meister et al. Broad-band efficiency calibration of ITER bolometer prototypes using Pt absorbers on SiN membranes. Rev. Sci. Instrum., 84(12), 2013.

[13] T. Nishitani et al. In-situ irradiation test of mica substrate bolometer at the JMTR reactor for the ITER diagnostics. Fusion Eng. Des., 63-64:437-441, 2002.

[14] A. Gusarov et al. Radiation stability of resistive bolometers intended for operation in the ITER environment. Fusion Eng. Des., 86(6-8):1200-1203, 2011.

[15] K. McCormick et al. New bolometry cameras for the JET Enhanced Performance Phase. Fusion Eng. Des., 74:679-683, 2005.

[16] H. Meister et al. Reliability issues for a bolometer detector for ITER at high operating temperatures. Rev. Sci. Instrum., 83:10D724, 2012.

[17] F. Forsberg et al. CMOS-Integrated Si/SiGe Quantum-Well Infrared Microbolometer Focal Plane Arrays Manufactured With Very Large-Scale Heterogeneous 3-D Integration. IEEE JOURNAL OF SELECTED TOPICS IN QUANTUM ELECTRONICS, 21(4):1-11, 2015. 